\title{
Structure of plastically compacting granular packings
}

\author{
Lina Uri, ${ }^{1, *}$ Thomas Walmann, ${ }^{1}$ Luc Alberts, ${ }^{2}$ Dag Kristian Dysthe, ${ }^{1}$ and Jens Feder ${ }^{1}$ \\ ${ }^{1}$ Physics of Geological Processes, University of Oslo, P.O Box 1048 Blindern, N-0316 Oslo, Norway \\ ${ }^{2}$ Department of Geotechnology, Delft University of Technology, Mijnbouwstraat 120, 2628 RX Delft, The Netherlands
}

(Received 5 September 2005; revised manuscript received 23 February 2006; published 1 May 2006)

\begin{abstract}
In this paper we present results of structural studies of compacting experimental systems of ductile grains in two and three dimensions. The high precision of our two-dimensional experiments enables a detailed study of the evolution of coordination numbers and local crystalline arrangements as a function of the packing fraction. The structure in both dimensions deviates considerably from that of hard disks and spheres, although geometrically, crystalline arrangements dominate on a local scale (in two dimensions). In three dimensions, the evolution of the coordination number is compared to experimental packings of hard and ductile grains from the literature. This comparison shows that the evolution of coordination number with packing fraction is not unique for ductile systems in general, but must depend on rheology and grain size.
\end{abstract}

DOI: 10.1103/PhysRevE.73.051301

PACS number(s): 45.70. $-\mathrm{n}, 61.43 .-\mathrm{j}, 61.66 .-\mathrm{f}$, 81.05.Rm

\section{INTRODUCTION}

The structure of monodisperse granular media is known to be very sensitive to the shape of the grains [1], grain interactions such as cohesion [2,3], and assembling procedure [4]. Packing fractions as low as $c=0.125$ have been found experimentally in three-dimensional (3D) systems with high graingrain attraction [5], whereas dense systems of spherical grains can be packed to $c>0.65$ [6] by a carefully selected tapping and assembling procedure.

Historically, the structure of dense granular media was studied during the 1960s and 1970s as a model of fluids and amorphous materials $[7,8]$. Since then, the complex properties of granular structures have been studied in dense elastic packings with much focus on compaction dynamics [9-12]. The densification of granular packings has a variety of applications, and in particular, it takes place in Nature during the slow compaction of sediments [13] and the fast event of a landslide. Granular compaction is also commonly studied in relation to pharmaceutical powders and metal industry [14]. In the compaction of sediments and powders, only the initial stages can be modeled by the compaction of hard elastic grain ensembles. When the geometrical structure is jammed [15] (i.e., no grain can be geometrically translated while all others remain fixed), at packing fractions $c \sim 0.64$, further compaction can only occur by grain deformation [13]. For the compaction of sediments and the isostatic pressing of metal powders, grains deform plastically, thus deformed regions are relaxed during the compaction. The final structural order and coordination number determine the compact's overall properties, such as its heat conduction [14]. Very little is known about the structure of plastically deforming grain ensembles, although they are extremely important for a range of industrially and naturally occurring compaction processes. The effect of grain ductility is thought to be especially large in two-dimensional monosized disk packings, as crystalline order dominates such systems of hard disks at low packing fractions $[16]$.

*Electronic address: 1.1.uri@fys.uio.no
Two-dimensional packings have been extensively studied from the theoretical point of view. In random sequential adsorption problems disks are placed one after another, without overlap, until the jammed state is reached in which no more particles can be placed without overlap [17]. Interestingly, the jamming packing fraction $c_{\infty}=0.547$ is approached asymptotically as $t^{1 / d}$, where $t$ is the number of attempts to place a disk without overlap, and $d$ is the spatial dimension, i.e., $d=2$ for the case studied in Ref. [17]. It was also shown that the pair-correlation function at jamming has a logarithmic divergence for touching disks. These results were consistent with observations of protein adsorption on solid surfaces. It is remarkable that the logarithmic divergence of the pair-correlation function $g(r \rightarrow 2 a) \sim \ln (r-2 a)$, and the approach to jamming $\left(c_{\infty}-c\right) \sim t^{-1 / d}$, is valid for any space dimension $d[18-20]$. By local rearrangements a more compact state with $c \simeq 0.772$ may be approached very slowly [21].

Recently it was shown that the "force trees" in twodimensional compressed packings of elastic disks exhibit a percolation type universal scaling [22]. Of course, the compaction of deformable grains will eventually reach the packing fraction $c=1$. Therefore an experimental study of the structure of two-dimensional and three-dimensional granular assemblies yields information on a highly relevant variable that makes such packings fall into a different universality class than the elastic packings.

In this paper, we present a series of experiments on ensembles of compacting ductile disk and sphere packings. High precision structural data (radial distribution function, coordination numbers, local geometry) were obtained from the two-dimensional experiments, and an "order-disorder" transition is seen at high packing fractions due to the ductile rheology of the grains. The average coordination number increases, surprisingly, in a linear manner with increasing packing fraction. In three dimensions the coordination number evolution is compared to that of other known packings from the literature, and possible compaction trajectories in coordination number versus packing fraction for ductile systems are discussed on the basis of geometrical restrictions. The following two sections (II and III) present the two- and 
three-dimensional systems. The discussion then follows in Sec. IV.

\section{THE TWO-DIMENSIONAL SYSTEM}

The two-dimensional (2D) system consisted of 1100 oriented cylinders, which were compacted at constant rate. From image analysis, the positions, coordination numbers, and packing fractions were found at regular intervals during the compaction.

\section{A. Experiment}

The 2D setup consisted of ductile cylindrical grains, which were stacked in a Hele-Shaw cell, and uniaxially compressed by a piston at a constant rate $v=1 \mathrm{~mm} / \mathrm{h}$. The grains were made of spaghetti, which had been boiled in water for $14.5 \mathrm{~min}$ in order to render them soft and deformable. The grains' resistance to deformation could be controlled by the boiling time. After boiling, the spaghetti was kept in a clear olive oil to avoid dehydration, then cut into cylinders of length $10 \mathrm{~mm}$, and carefully stacked in the container (HeleShaw cell). Two sizes of grains were used, but in different sections of the model. For simplicity, only the lower section of nearly 600 monodisperse grains was used in this analysis. These grains had (initial) diameters of $d=(2.8 \pm 0.1) \mathrm{mm}$.

The width and height of the initial packing was $w=83 \mathrm{~mm}$ and $h=92 \mathrm{~mm}$, corresponding to 29 and 33 grain diameters, respectively. The initial height of the 600 lower grains was $55 \mathrm{~mm}$, or 20 grain diameters. During the stacking, olive oil was constantly added to the container so that the grains were immersed in oil at all times. The oil, apart from preventing dehydration of the grains, also lubricated the walls so that friction did not restrain the compaction. The depth of the Hele-Shaw cell was $11-12 \mathrm{~mm}$, to ensure a channel at the back of the stacked grains for the oil to escape through. The oil thus did not affect the compaction mechanically. Figure 1(a) illustrates the setup of the 2D experiment, and Fig. 2 contains a closeup of a region in the first image. Pictures were taken every $20 \mathrm{~min}$, and a total of 47 pictures were taken as the system compacted at a speed of $v=1 \mathrm{~mm} / \mathrm{h}$ from an initial packing fraction of $c=0.89$ to the final $c=0.99$. The camera was an AstroCam Capella, LSR Life Science Resources, UK, with $3000 \times 2000$ pixels, 14 bit resolution. Due to reflections in the setup the useful dynamic range that described grains and pores was reduced to slightly less than 10 bit during the experiment. The spatial resolution was 23 pixels per $\mathrm{mm}$.

\section{B. Analysis (2D)}

The geometrical center position was obtained for all grains by image analysis. In the first image, i.e., the image taken immediately after compaction started, the center of mass was found by the following procedure: considering circles around a particular pixel, the radius of the circle that contained 5 pixels of intensity below a certain threshold was taken to be the shortest distance to the pore space from this particular pixel. The pixel within a grain with the largest distance to the pore space was taken to be the center position

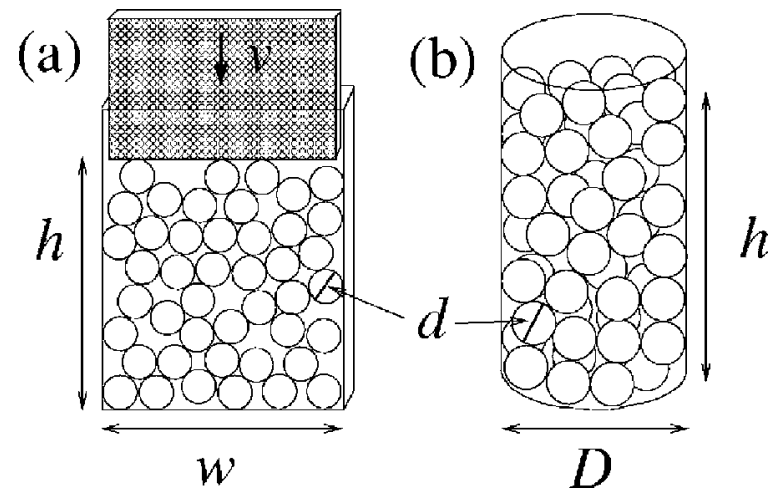

FIG. 1. Schematic illustrations of the experimental setups. $h$ is the filling height and $d$ is the grain diameter in both 2D and 3D. (a) The quasi-two-dimensional arrangement. $w$ is the width of the container, and the piston is driven at constant velocity $v$. The grains were cylinders made from spaghetti, and stacked such that their length was in the depth of the container. When viewed from the front of the container only the circular cross section of the grains were seen, of diameter $d$. (b) The three-dimensional cylindrical ensemble, consisting of spherical grains of diameter $d$ made of Play Doh. The inner container diameter is $D$.

of the first image, see Fig. 2 for an illustration. None of the disks were much deformed at this stage, so looking for pore space in circular sections accurately determined the center position to a resolution of a fraction of a pixel for most grains. However, due to reflected light from the Hele-Shaw cell, some positions were only determined within a few pixels resolution. The center of mass positions found in the first image were traced by pattern recognition (normalized cross correlation function [23]) in all the following images, to a resolution of 1 pixel.

The grains were essentially incompressible. During compaction, the average cross-sectional area $A$ of each grain decreased $6 \%$, thus the grains elongated in the direction perpendicular to the image plane. The development of the crosssectional area was found manually for ten specific grains in

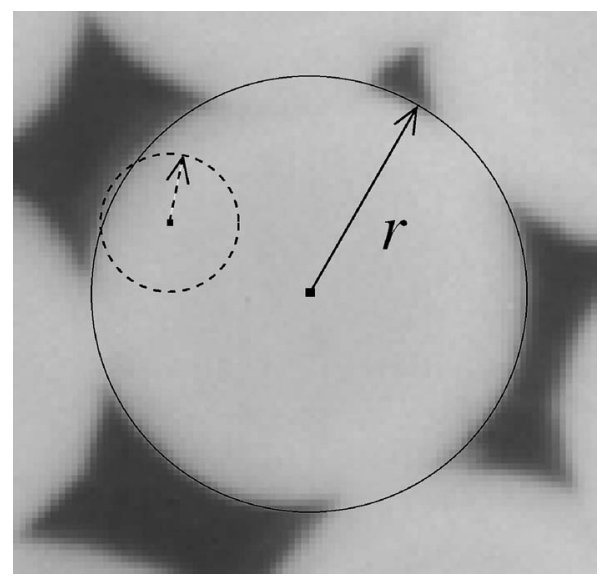

FIG. 2. Closeup of a grain in the first image. The procedure of finding the grain center in the first image is illustrated: the two circles represent the largest distance from their center pixels at which a maximum of five "dark" pixels are found (see text for details). 


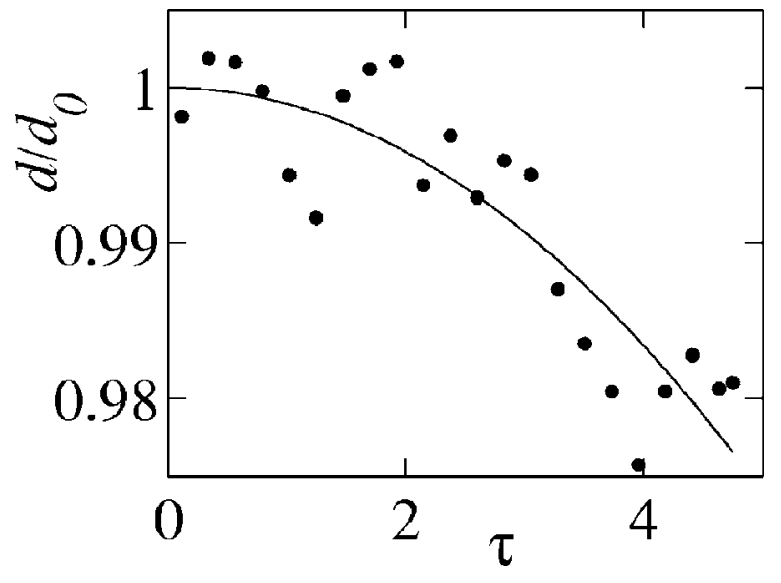

FIG. 3. The normalized effective grain diameter $d / d_{0}$ (line) as a function of dimensionless time $\tau=t v / d_{0}$. (•) Grain diameters $d=\sqrt{4 A / \pi}$ calculated from the average of the measured area $A$ of two grains.

the first and last image. Of these ten grains, two representative grains were selected, and their areas were found (also manually) in every second image throughout the experiment. A best fit was made to the developing average area, as $A(\tau)=a\left[1-c(\tau)^{2}\right]$, where $\tau=t v / d_{0}$ is dimensionless time, $a=3.6 \times 10^{3}$ square pixels, $c=0.0021, v$ is piston velocity, and $d_{0}$ is the initial diameter. The effective grain diameter $d$ in each image was then found as $d(\tau)=\sqrt{4 A(\tau) / \pi}$. Figure 3 shows the grain diameter, calculated from the average area of each image, and the effective diameter $d$ (line) as functions of dimensionless time $\tau$. By using the effective diameter in all the analysis, the decreasing cross-sectional area does not affect the geometry and structural evolution during the compaction. Thus, the packing fractions and radial distribution functions can be compared between images independent of the actual grain diameters.

\section{Packing fractions}

The packing fraction $c$ of the 2D system was obtained by Voronoi analysis of the position data from each image. Only disks at a distance of more than one grain diameter from any boundary were used in the calculation to avoid unbounded [24] Voronoi cells. The area $A_{v i}$ of the Voronoi cell for each disk center $i$ was found, and as each such cell contains one disk, the local packing fraction $c_{i}$ was given by

$$
c_{i}=\pi d^{2} / 4 A_{v i},
$$

where $d$ is the average diameter of a grain in the image in question. The Voronoi tessellation of a region around a certain grain is shown for the first and last (dashed) image in Fig. 4(a), with grain centers marked as bullets (first image) and circles (last image). The motion relative to the central grain is indicated by lines between the grain center positions in the first and last image. Figure 4(b) shows the cumulative distribution $P(r)$ of distances between touching grains for the first (curve A) and last (curve B) images. The overall packing fraction based on the Voronoi cell division was $c=\left\langle c_{i}\right\rangle$. The 2D compaction spanned packing fractions from $c=0.89$ to $c=0.99$.
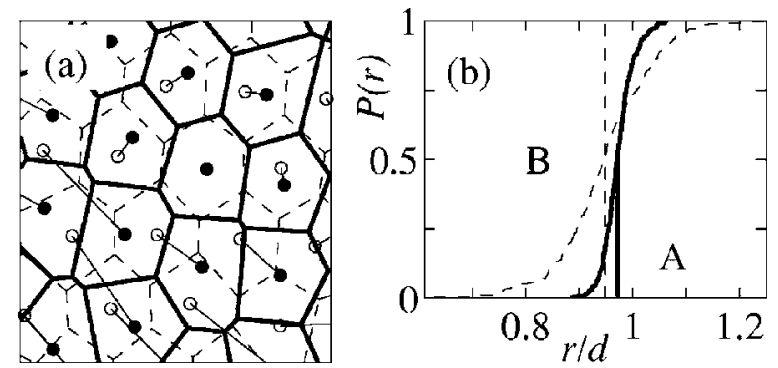

FIG. 4. (a) An example of the Voronoi structure in a region of the first and last (dashed) images, used in obtaining the local packing fractions $c_{i}$. The tessellation of the last image was translated so that the center of one cell lie undistorted. The compaction direction is downward. The cumulative distributions of touching neighbor distances are shown in (b), for the first (A) and last (B, dashed) images. The average value of distances between touching neighbors is indicated by vertical lines for the first and last (dashed) image.

\section{Radial distribution function}

The radial distribution function (RDF) $g(r)$ is defined by $g(r)=\rho(r) /\langle\rho\rangle$, where $\langle\rho\rangle=N / V$ is the average number density of grain centers in a container of area $V$, and $\rho(r)$ is the average number density as a function of distance $r$ from a grain center. The expression for the number density as measured from grain number $i$ is

$$
\begin{gathered}
\rho_{i}(r)=\frac{N_{i}(r)}{V_{n}(r)}, \\
N_{i}(r)=\sum_{j \neq i} \delta\left(r_{i j}=r\right) .
\end{gathered}
$$

Here, $V_{n}(r)=2 \pi r$ is the circumference of a circle of radius $r$, and $N_{i}(r)$ is the number of grain centers at distance $r$ from grain $i . r_{i j}$ is the distance between grains $i$ and $j$, and $\delta$ is the Kronecker delta, $\delta=1$ for $r_{i j}=r$, and otherwise zero. The average $\rho_{i}(r)$ over $N$ grains gives

$$
\rho(r)=\frac{1}{N} \sum_{i=1}^{N} \rho_{i}(r)
$$

thus

$$
g(r)=\frac{1}{N\langle\rho\rangle V_{n}(r)} \sum_{i} N_{i}(r),
$$

where $i$ is summed over all grain centers of the sample. In a finite system the expression for $g(r)$ should be replaced by

$$
g(r)=\frac{1}{N\langle\rho\rangle V_{n}(r, d r)} \sum_{i} N_{i}(r \in[r-d r / 2, r+d r / 2]),
$$

where $d r$ is the width of the shell, its size depending on the number of grains in the system and/or the uncertainty of the position measurements. Perturbations to $d r$ should not affect $g(r)$ when the proper value of $d r$ is chosen, as the dependence of $N_{i}$ and $V_{n}$ on $d r$ should cancel in the expression of $g(r)$. The expression for the circular shell becomes $V_{n}(r, d r)=\pi\left[(r+d r / 2)^{2}-(r-d r / 2)^{2}\right]=2 \pi r d r$. 
Noting that the sum in Eq. (6) over $N_{i}$ equals twice the number of distances $n(r, d r)$ of lengths $r \in[r-d r / 2, r$ $+d r / 2]$ in the ensemble, $\Sigma_{i} N_{i}(r, d r)=2 n(r, d r)$, the expression for the RDF of a finite packing becomes

$$
g(r)=2 n(r, d r) F(r) /\left[N\langle\rho\rangle^{2} V_{n}(r, d r)\right],
$$

where $F(r)$ is a finite size correction for the boundaries, as discussed below.

When Eq. (7) is used without the normalization $F(r)$, the boundaries of the ensembles introduce finite size effects to the RDF of a small system. These finite size effects result not only because the structure along the boundaries differs from the interior structure, but because the normalization over circular shells of areas $2 \pi r d r$ includes regions outside of the ensemble. To avoid the latter source of errors, the normalization function $F(r)$ is introduced based on the specific rectangular geometry of the ensemble. $F(r)$ is found as follows: In an infinite system, a grain in a position $\boldsymbol{R}=(x, y)$ would be surrounded by a circular shell $2 \pi r d r$ independent of its position. In the finite system of a container, whether all of the circular shell lies within the container depends on the radius $r$ and the grain's position $\boldsymbol{R}$. The fraction of circular shells of radius $r$ that partly lie outside of the container increases with $r$ and is a measure of the error done by disregarding the boundaries. Let the area of the container be $V$. The integral over $V$ of all circular shell circumferences $2 \pi r$ with center positions inside the area of the container is $2 \pi r V$. Only a part of this integral represents circumferences that lie inside the container, thus in normalizing the RDF of a finite system one should use this fraction instead of the $2 \pi r V$. A normalization function $F(r)$ can be defined as the ratio of the integrals of circumferences in the infinite case to the finite case

$$
F(r)=\frac{\int_{V} 2 \pi r d^{2} \boldsymbol{R}}{\int_{V} A(r, \boldsymbol{R}) d^{2} \boldsymbol{R}} .
$$

$A(r, \boldsymbol{R})$ is the fraction of the circular shell of radius $r$ centered at $\boldsymbol{R}$ that lies inside the container. For a rectangular container of width $w$ and height $h$ the normalization function is

$$
\begin{aligned}
F(r)= & \pi w h[\pi(w-2 r)(h-2 r)+(\pi-1)(w+h-4 r) \\
& +r(3 \pi / 2-2)]^{-1} .
\end{aligned}
$$

This correction procedure was previously used by Mason [25] for a different geometry (3D spherical ensemble).

\section{Coordination numbers}

The coordination numbers $k$ (number of contacts per grain) were found from further image analysis; a Delaunay triangulation based on the position data was used to obtain the set of nearest neighbors of each grain. Each Delaunay neighbor connection was then investigated by a thresholding procedure to establish whether it was a touching neighbor: The intensity values of the array of pixels that formed the shortest path between grain centers $i$ and $j$ was considered. A

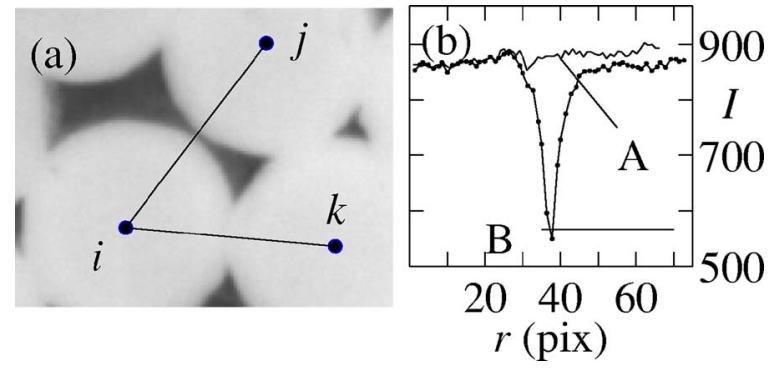

FIG. 5. (Color online) The procedure of finding touching neighbors was based on the intensity values of the direct line between grain centers. (a) shows a small region of the initial compaction state, where $i, j$, and $k$ are grain centers. The lines $i-j$ and $i-k$ are the direct lines between the centers, of which the intensities are plotted in (b) as curves A and B, respectively. The dip shown in curve B was typical when grains did not touch.

distinct dip in the values was observed for grains not touching, as shown for path A in Fig. 5, while for touching grains no such dip was observed. All grains having arrays with intensity dips below a ratio $2 / 3$ of the local typical grain intensity value were considered touching. The particular choice of ratio did not matter as the procedure was robust for a range of sensible values, which was confirmed by visual inspection. The threshold value had to relate to the local typical grain intensity value because of somewhat uneven illumination over the sample. Figure 5 illustrates the procedure. Grains $i$ and $j$, as seen in Fig. 5(a), are not touching as the minimum value of their center-to-center intensity plot is below $I=850(2 / 3)=567$, as seen in (b). The typical intensity difference between (large) pore space and a grain was nearly 10 bit, while for small pores [such as the gap in Fig. 5(a)], it was reduced but still larger than 8 bit. The coordination number distribution $P(k)$ was found with this procedure for all the images.

\section{Results (2D)}

The radial distribution functions for the first and last stages of the experiment are shown in Fig. 6. The structure at $c=0.89$ shows many of the characteristic peaks of a triangular packing of disks, except that the peaks are broadened and shifted to shorter distances than those of a crystalline packing. Crystalline correlations occurring in triangular lattices of hard disks at $r / d=(1, \sqrt{3}, 2, \sqrt{7}, 3)$ are shifted to $r / d=(0.958,1.695,1.915,2.580,2.875)$. In the RDF of the more compacted system of $c=0.99$ the peak near $r / d=3$ has vanished, and the double peak near $\sqrt{3} \simeq 1.7$ has become smoother and broader. Also the first peak $(r / d=1)$ is seen to broaden considerably as many neighbor distances at this stage are less than one effective grain diameter. The inset of Fig. 6 shows the finite size correction function $F(r)$ for the first image, which is practically identical to the correction in the last image.

The geometrical structures associated with the peaks at $r / d=1.695$ and $r / d=1.915$ in the RDF are illustrated in Fig. 7 by a conditional three-point correlation function [26]. Figure 7(a) was generated by identifying all grain centers at 


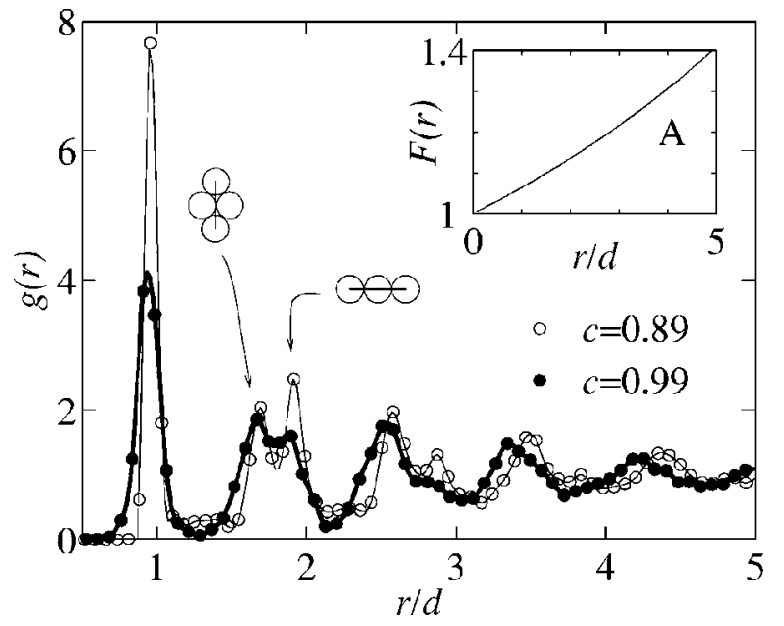

FIG. 6. The radial distribution function $g(r)$ as a function of particle separation $r$ in the ductile 2D experiment, for packing fractions $c=0.89(\bigcirc)$ and $c=0.99(\bullet$, bold line). The curves connecting the data points are produced by splines. The normalization function for the 2D setup $F(r)$, Eq. (9), is shown in the upper right corner (curve A) for the distances $r / d \in[0,5]$. The typical geometrical configurations that contribute to the peaks at $r / d \in[1.695 \pm 0.05]$ and $r / d \in[1.915 \pm 0.05]$ are illustrated.

distances $r / d \in[1.695 \pm 0.05]$ to each other in the packing, aligning each pair of such centers along the horizontal axis, and plotting the surrounding grain centers in the corresponding positions. The two fixed grain positions are shown on the horizontal axis at the center of the figure, while the surrounding intensity structure visualizes the probability of having a third grain in any position relative to the two fixed ones. The intensity was normalized by the number of pairs contributing to the plot. Figure 7(b) is generated in the same manner as (a), but for grains at a distance of $r / d \in[1.915 \pm 0.05]$ to each other. The upper half of (a) and (b) shows the probability distribution for the initial stage of the compaction $(c=0.89)$, while the lower part shows the distribution at $c=0.99$. Black represents a high probability of the third grain and white represents zero probability. The black circles mark the distance of one diameter from the two fixed grain positions. The intensity pattern suggests a regular structure with obvious symmetries in the packing. At first glance, the structure

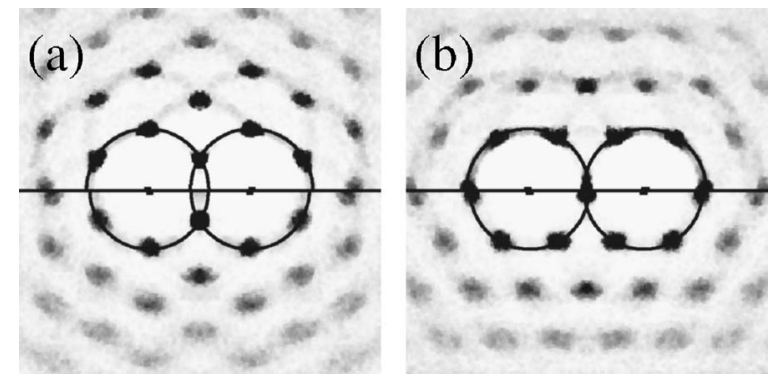

FIG. 7. Visual illustration of the probability of a third grain position for two grains at a given distance (a) $r=1.645-1.745 d$ and (b) $r=1.865-1.965 d$. The upper parts of the plots show the probability distribution at $c=0.89$, while the lower give the distributions at $c=0.99$.

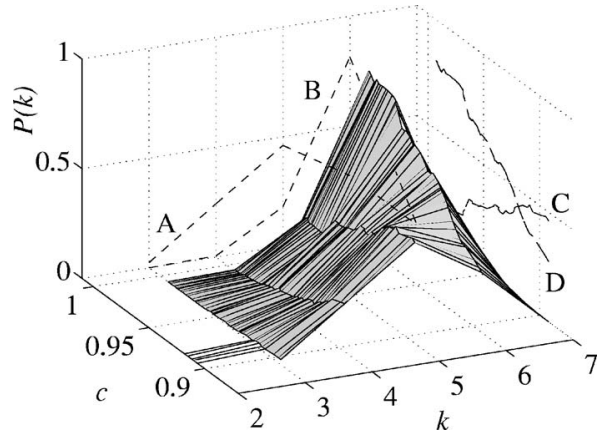

FIG. 8. The distribution $P(k)$ of coordination numbers $k$ as function of packing fraction $c$ in two dimensions. Curves A and B are projections of $P(k)$ at $c=0.89$ and 0.99 , respectively. Curves $\mathrm{C}$, for $P(5)$, and $\mathrm{D}$, for $P(6)$, are developments of $P(5)$ and $P(6)$ with increasing $c$.

might seem hexagonal, but this is due to the alignment of the two fixed centers; The underlying structure is triangular. Although the intensity plot of Fig. 7(a) does not prove the presence of the four-point configuration illustrated in Fig. 6, closer inspection of the configurations contributing to this peak (at $r / d=1.695$ ) in the RDF shows that this configuration is dominant for $r / d=1.695$. Similarly, the configuration of three grains in a row is seen to contribute most to the peak at $r / d=1.915$ of the RDF, although the center grain is often slightly misplaced along the axis. The lower part of Fig. 7(a) contains a small probability of a third grain in between the two fixed grains (barely visible in the figure), which is not seen in the upper part $(c=0.89)$. This very compressed alignment of three grains in a row emphasizes the role of ductility of the grains, as this configuration is impossible in packings of hard disks.

The cumulative distribution $P(r)$ of distances between touching grains is shown in Fig. 4(b). The average distance between touching grains decreases from the first to the last image, while the width of the distribution increases. The touching grains at distances larger than a grain diameter are grains aligned perpendicular to the compaction direction, thus the ellipticity of grains increases, especially in the last compaction stage.

The coordination number distribution is shown in Fig. 8 as a three-dimensional plot of the distribution $P(k)$ as function of coordination numbers $k$ and packing fraction $c$. The distribution is broad at $c=0.89$, and then narrows and shifts to higher coordination numbers with increasing packing fraction. At packing fractions $c=0.906-0.918$ a few cases of grains with only two contacts are observed, but only one or two such grains are present at each packing fraction. Curves A and B in Fig. 8 are projections of the first and last distributions of the experiment, respectively. Curves $\mathrm{C}$ and $\mathrm{D}$ are projections of the fractions of five and six coordination numbers with increasing packing fraction.

Figure 9 shows the average coordination number $\langle k\rangle$ as function of packing fraction in the two-dimensional experiment. The circle at coordinates $(1,6)$ represents the end point for 2D systems, as a space filling structure (Voronoi cells) in 2D must have six neighbors on average [27]. Also shown are the data for two crystalline structures, the square lattice and 


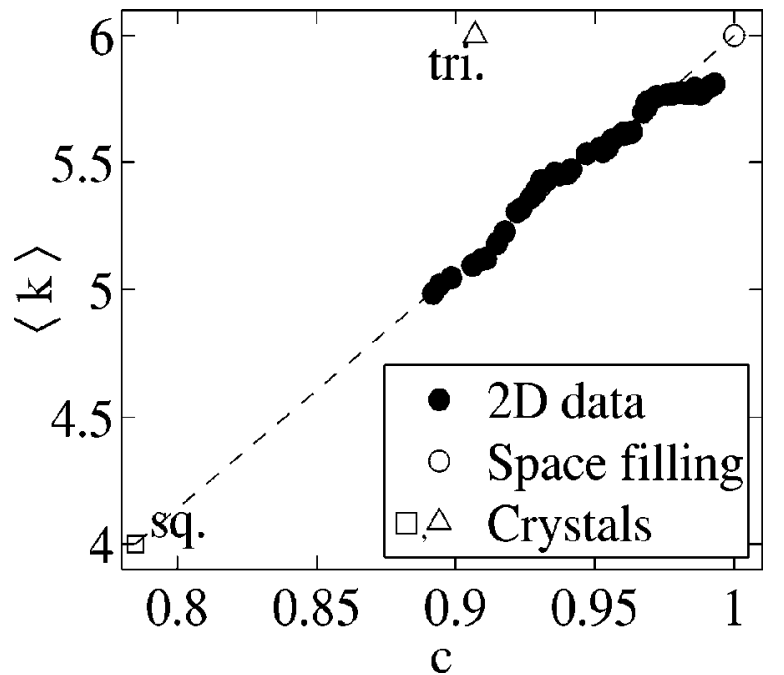

FIG. 9. Mean coordination numbers $\langle k\rangle$ as a function of packing fraction $c$ for the two-dimensional ductile experiment. The circle represents the $2 \mathrm{D}$ space filling structure.

the triangular lattice. We observe that the evolution of the average coordination number in the ductile packing closely follows a straight line between the square lattice and the space filling Voronoi structure.

\section{THE THREE-DIMENSIONAL SYSTEMS}

The three-dimensional (3D) systems analyzed here are six experimental systems of compacting ductile grain ensembles. These are compared to Finney's experiment [7] on a random close packed ensemble of hard spheres $(c=0.6366)$, from which the positions were kindly made available to us. This ensemble contained nearly 8000 grains in a square box, and the positions of the grains were measured to a precision of $0.2 \%$ of the grain diameter. A numerical model of a compacting grain ensemble is also presented, giving possible trajectories for compaction between packing fractions $c=(0.50-0.64)$.

\section{A. Experiments}

The 3D experimental setup consisted of $N$ ductile grains poured into a cylindrical Plexiglas container, approximately ten at a time, to a height $h$. The set of grains were then left to compact under gravity for a time $t$, or were mechanically compacted to a specific packing fraction. A lid covered the top of the cylinder during the experiment to avoid dehydration, as the grains contain water and other volatile chemicals. Figure 1(b) illustrates the setup of the 3D experiments. The grains were prepared from Play-Doh (Hasbro Int., Inc., UK) to spheres of diameters $d=(8.8 \pm 0.2) \mathrm{mm}$. The material is viscous for shear rates in the range $\left(10^{-3}-10^{-6}\right) / \mathrm{s}$, with a viscosity of $3 \times 10^{5} \mathrm{Pas}$, as measured in rotational tests (Paar-Physica MCR 300, rheometer). Except from the first few minutes and the very end of an experiment, the strain rate was within this regime. Strain rates down to $10^{-7} / \mathrm{s}$ were common after a week's compaction, but shear tests could not be performed at such low rates, and the material properties for these rates are not known.

Each experimental system was disassembled grain by grain while measuring positions and/or coordination numbers of the ensemble. The position coordinates $(x, y, z)$ of the grains' top point were established with a mechanical arm (MicroScribe) to an estimated resolution of $0.5 \mathrm{~mm}(6 \%$ of grain diameter), limited by the difficulty of establishing the center of the grains' top surface. The number of touching neighbors (coordination number) was also counted for each grain during the disassemblage. To ease this procedure, grains of five different colors were used, as grains of different colors left marks on each other in contacting areas. Before a grain was removed, the visibly contacting neighbors were counted. The grain was then removed, and any formerly hidden contacts became visible due to grain deformation and/or discoloring. As a grain was removed, all its contacting grains that remained in the ensemble were carefully marked with a felt pen. Thus, the coordination number of a grain consisted of the number of marks on its upper surface, plus the number of visible and hidden contacts established during the removal of the grain itself. The number of contacts that each grain had to the cylinder wall and/or floor was also recorded.

Six different three-dimensional ensembles were studied: Two of them with 2200 grains in a cylinder of diameter $D=130 \mathrm{~mm}$, the rest with 150 grains in cylinders of diameter $D=32 \mathrm{~mm}$. The four small systems were prepared simultaneously, and left to compact for different amounts of time before each was disassembled and the positions and coordination numbers of each system measured. The resulting packing fractions were $c=0.64,0.64,0.66$, and $0.68 \pm 0.01$. The small system size was desirable in order to avoid considerable compaction during the disassemblage for the short compaction times. One of the big systems was left to compact for $145 \mathrm{~h}$ before it was disassembled, measuring the positions and coordination numbers of the grains. The packing fraction went from 0.57 (based on filling height) to 0.701 during the compaction in this ensemble. Due to a calibration error with the mechanical arm, only the positions of 787 grains in the mid to lower part of the cylinder were successfully measured, while the coordination number was established for 1169 grains. The second large system was compacted to a packing fraction of $c=0.75$ by gently pushing a piston from the top of the cylinder. Compacting the system to such high packing fractions would otherwise have required months of gravitational compaction. Only the coordination numbers were measured in this ensemble, for 839 central grains.

\section{B. The Finney ensemble}

Finney [7] carefully measured the positions of nearly 8000 monodisperse spheres in a box of square cross section. The spheres were steel ball bearings of diameters $1 / 4$ inch, which positions were measured to $0.2 \%$ of the grain diameter. The central 817 grains were used in this analysis, as these grains had a minimum distance of five grain diameters to any boundary, thus no corrections to the RDF was neces- 
sary to $r / d=5$. Based on this selection, the radial distribution function and coordination numbers were found.

\section{Numerical elastic compaction model}

A numerical model "Rampage," see Ref. [28], was used to simulate granular packings of elastic, spherical grains in a gravitational field. This model was originally developed for the modeling of sediment compaction, and is described in detail in Ref. [28]. One thousand monodisperse spherical grains of diameter $d$ were randomly distributed at packing fraction 0.5 in a cubic volume with a soft repulsive potential at the bottom wall and with periodic boundary conditions applied in the horizontal plane. No considerations about overlapping particles $\left(r_{i j}<d\right.$, where $r_{i j}$ is the interparticle distance) were done during this initial filling procedure. Compaction of the ensemble proceeded iteratively by displacing particles until all particles were gravitationally stable with minimum overlap. The scheme was not energy conserving in that no acceleration or velocity was considered, but at each iteration the length of displacement of a particle was proportional to the sum of forces (interparticle elastic, gravitational, and tapping "forces"). The interparticle forces were taken to be proportional to $d-r_{i j}$ when $r_{i j}<d$ and zero otherwise, and the gravitational force was constant downward. After reaching a gravitationally stable ensemble with minimum overlap further compaction was obtained by "tapping": an incremental $(<0.001 h)$ vertical repositioning downward of each particle (at height $h$ ), followed by the same procedure of reducing overlaps and reaching gravitational equilibrium. Packing fractions of stable numerical ensembles were in the range $c=0.55-0.64$, which is the typical range of randomly packed spheres. The model reproduces statistically Finney's ensemble at $c=0.636$ with respect to the RDF, the coordination number distribution and the distribution of contact angles, see Ref. [28]. The numerical model was not used as a model for the ductile experimental systems, but as a model of hard sphere packings in order to obtain the average coordination number as a function of packing fraction.

\section{Analysis (3D)}

\section{Packing fractions}

In three dimensions, the packing fractions were computed from the position measurements for all the cases in which these were available, which were most of the ductile experiments, the Finney ensemble, and the numerically generated ensembles. Boxes of variable square cross section $l^{2}$ and height $h$ equal to the height of the packing were centered in the granular ensemble for calculation of the packing fraction. For each of these boxes $j$, the packing fraction was $c_{j}(l)=N_{j} V_{g} / V_{b j}$, where $N_{j}$ is the number of grains contained in the box, $V_{g}=\pi d^{3} / 6$ is the average volume of a grain, and $V_{b j}=l^{2} h$ is the volume of the box. Grains that partially intruded the box, i.e., when their center position was less than one grain diameter from the box boundary, also contributed to the number of grains in the box. The overall packing fraction $c$ of an ensemble was obtained by averaging over $n=10$ boxes in distances $0.5-1.5$ grain diameters $d$ from the boundary

$$
c=\frac{1}{(n+1)} \sum_{j=0}^{n} c_{j}\left(l_{0}+j \Delta l\right),
$$

where $l_{0}$ is the side of the box in a distance 1.5 grain diameters from any boundary, and $\Delta l=d / n$. In the numerical model, the total volume of two touching grains was smaller than two grain volumes if the grains overlapped. The overlap volume was not assumed to be transported to the pore volume, as this would effectively change the structure of the packing, thus the calculation of the packing fraction must account for the excess overlap volumes in the packing. This was done by reducing the sphere radii by the mean overlap distance in the packing, thus reducing the volume of the grains before calculating $c$.

\section{Radial distribution function}

The radial distribution function in three dimensions is given by Eqs. (6) and (7), but with $V_{n}$ as the volume of a spherical shell of radius $r$ and width $d r$ in 3D: $V_{n}=4 \pi\left[(r+d r / 2)^{3}-(r-d r / 2)^{3}\right] / 3=4 \pi r^{2} d r+\pi(d r)^{3} / 3$.

Equation (6) was used in the calculation of the RDF in the Finney ensemble, as an ensemble average was made only over the central 817 grain positions to avoid boundary effects. These grains were all in a minimum distance of five grain diameters from any boundary, thus the RDF is not affected by boundaries up to $r / d=5$. In order to calculate the RDFs of the large ductile ensemble $(c=0.70)$, Eq. (7) was used, as the statistics of the whole ensemble was needed for a sufficiently detailed RDF to be found. A finite size correction function $F(r)$ was introduced for the ductile ensemble in the same manner as for the 2D system, Eq. (8). In 3D, $A(r, \boldsymbol{R})$ is the fraction of the spherical shell area [radius $r$, center position at $\boldsymbol{R}=(x, y)$, height $z$ ] which is inside the cylindrical container. The resulting correction function is

$$
F(r)=\frac{4 \pi^{2} r^{2} R^{2} h}{\int_{0}^{\min (h, r)}\left(I_{1}-I_{2}\right) d z},
$$

where

$$
\begin{gathered}
I_{1}=2 \pi^{2} r(h-z) R^{2} \\
I_{2}=-2 \int_{0}^{1} 4 \pi r(h-z)(R-u x) \arccos \left[\frac{2 x R-u\left(x^{2}-1\right)}{2(R-u)}\right] d x
\end{gathered}
$$

and $u=\sqrt{r^{2}-z^{2}} \cdot F(r)$ is presented in the inset of Fig. 10. $R$ is here the length of $\boldsymbol{R}$, i.e., the radial distance of a grain center from the cylinder axis. The width of the spherical shells were chosen so that the standard error of distribution from the mean was less than $2 \%$ of the RDF, for the second peak. This gave the following resolutions of the RDFs; $d r / d=0.1$ (ductile ensemble) and $d r / d=0.02$ (Finney). 


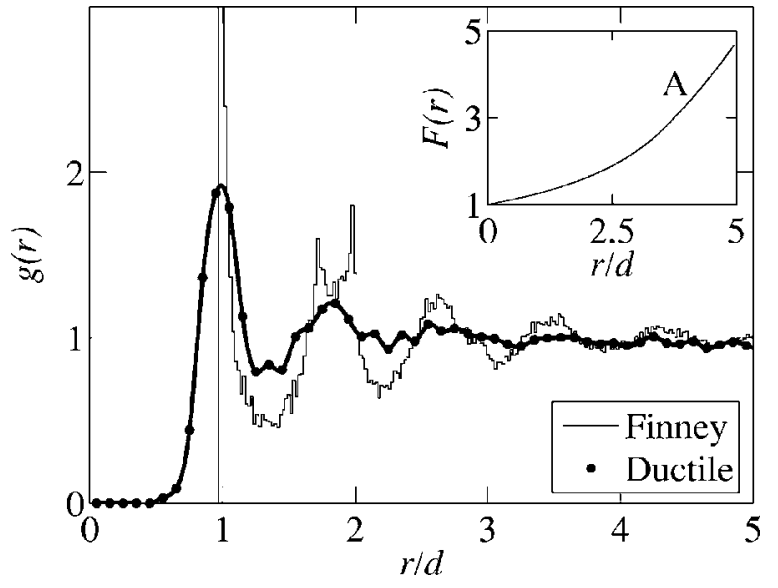

FIG. 10. The radial distribution functions for an ensemble of ductile grains $(\bullet$, bold line) and for the Finney ensemble of dry, hard grains (thin line). The bold line was obtained by splines to the data. The inset shows the normalization function $F(r)$, see Eq. (11) (curve A) used to correct for the 3D finite size effects for the ductile system, in the region $r / d \in[0,5]$.

\section{Coordination numbers}

Coordination numbers in three dimensions were found experimentally for the ductile ensembles, as described in Sec. III A. As the grains in contact to the walls were known, the distributions of coordination numbers for the ductile ensembles are based only on the internal grains. In the second of the large ensembles $(c=0.75)$, only grains at a minimum distance of three grain diameters from any boundary were included. For the Rampage and Finney ensembles, the coordination numbers were based on the position data. In the Rampage ensembles grains in a distance less than one grain diameter apart were considered touching, as the model allows small overlaps between grains. In the analysis of the Finney ensemble, grains were considered touching if the center to center distance $r$ was less than 1.02 grain diameters. The choice of this distance was based on the average contact number obtained when different $r$ 's were assumed for touching neighbors: If grains were assumed touching only when their center to center distance was $1 d$ or less, $\langle k\rangle=0.9 .\langle k\rangle$ rapidly increased as the assumed distance for touching grains increased, and at $1.02 d$, the average coordination number was 6.72. Also, at $1.02 d$, all grains (except for one) had at least three neighboring grains at distances closer or equal to $r / d=1.02$, which is the stability criterion in $3 \mathrm{D}$ for grains shielded by granular bridges.

\section{E. Results (3D)}

To calculate RDFs, the center positions of the grains must be known, whereas in the $3 \mathrm{D}$ ductile experiments the positions of the top points of the grains were measured. Although the top points are not the shifted positions of the grain centers, they were used in calculating the RDF, as they represent correlations between specific points in the ensemble in the same way that the center positions do. Also, it should be directly comparable to the RDFs of hard granular ensembles, as in these the top positions are truly linear translations of the

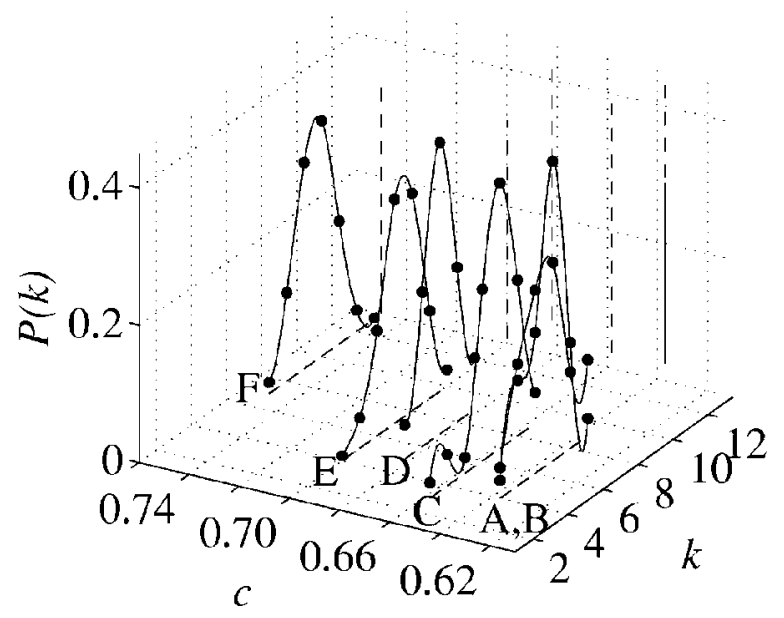

FIG. 11. The distribution of coordination numbers for the different ductile ensembles, as a function of packing fraction. The curves $\mathrm{A}-\mathrm{F}$ are splines to the data points of the packings at packing fractions $c=0.64,0.64,0.66,0.68,0.70$, and 0.75 , respectively.

center positions. The detailed structure of an ensemble can only appear in the RDF if there is a sufficient amount of grains in the ensemble. Only the large ductile system contains enough grains to capture the main features of the RDF, thus unfortunately, we can only compare the ductile system at a packing fraction of $c=0.70$ to the Finney ensemble $(c=0.636)$, and only at a resolution of $d r=0.1 d$. The normalized RDF of the ductile ensemble is shown in Fig. 10, as is also the RDF of Finney's ensemble for dry granular media. From the figure, we see that the ductile ensemble is much less ordered than the hard granular ensemble, as all peaks and valleys in the RDF are small compared to the Finney RDF. We also note that the RDF of the ductile packing has a value for distances smaller than one grain diameter, which is not surprising, as this reflects the grains' ability to deform and thus obtain distances closer than one grain diameter. We see fewer clear peaks for the ductile ensemble than for the Finney ensemble, as deformed grains broaden the peaks. The split second peak in the RDF of the Finney ensemble is not present in the ductile ensemble.

The coordination number distributions for the ductile systems are presented in Fig. 11. The distributions shift to higher coordination numbers as the density increases, as is to be expected. One interesting observation is that two of the distributions for the small ensembles contain grains of a coordination number as low as three. This is possible for grains that are shielded by granular bridges, which are also found in the Finney and Rampage packings to a similar degree $(1.6 \%$ of the internal grains). Due to the cohesion between the ductile grains, the effect of granular bridging was expected to be more dominant in the looser ductile structures than in the Finney and Rampage structures, which is not the case. For the denser ductile structures $(c>0.64)$ all grains have coordination numbers larger than 3 , but the width of the distributions remains the same within $4 \%$. Figure 12 shows the mean coordination numbers as a function of packing fraction for the ensembles presented in this paper, and also for a few cases from the literature. The compaction of the ductile grain 


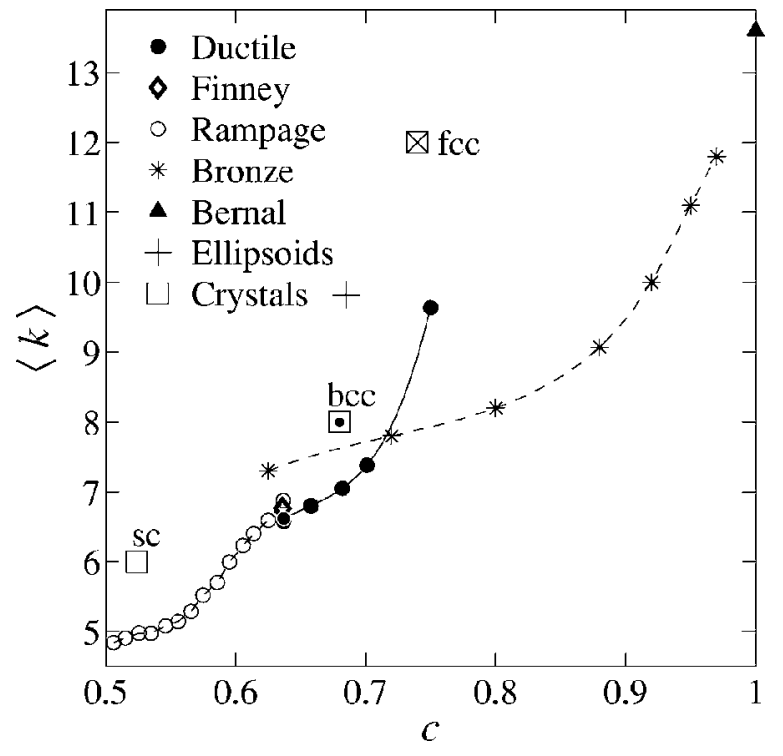

FIG. 12. Mean coordination numbers $\langle k\rangle$ as a function of packing fraction $c$ for several kinds of packings: The ductile ensembles $(\bullet)$, the Finney ensemble $(\diamond)$, Rampage ensembles $(\bigcirc)$, compacting bronze powder [14] $(*)$, compacted plasticine [29] ( $\mathbf{\Delta})$, hard ellipsoid ensemble [1] $(+)$. The data for some crystalline structures of hard spheres are shown for reference: Simple cubic $(\square)$, body centered cubic $(\square)$, and face centered cubic $(\otimes)$.

ensembles (filled circles) start in the neighborhood of the random close packed Finney ensemble (diamond), and then $\langle k\rangle$ increases with $c$. The first five points on this curve were obtained from gravitationally compacted ensembles, thus their compaction process is the same. The last point, at $c=0.75$, was obtained by mechanical compaction of a piston, and therefore does not necessarily represent the natural evolution of the gravitational ensembles. The numerical model (Rampage) evolved along the curve represented by open circles in Fig. 12, during one simulation. It ends up also in the neighborhood of the Finney ensemble. Note that the trajectory of the Rampage packing does not seem to be continued by the ductile ensembles, as the curves have different slopes at $c=0.64$. Another ductile ensemble from the literature is presented in the figure (asterisks): Fischmeister et al. [14] conducted compaction experiments on bronze powder by die compression at various pressures. The compacted ensemble was forced open after compression, and the number of contacts and porosity measured. For the smallest packing fraction, the powder was sintered in order to mark the contact points of a noncompressed ensemble. The data at higher packing fractions were compacted at pressures of $0.2,0.4$, $0.6,0.8$, and $1.0 \mathrm{GPa}$, respectively. The powder had an initial size distribution of 250-315 $\mu \mathrm{m}$. The packing of hard ellipsoids [1] is marked as a plus sign in Fig. 12, and the crystalline ensembles of simple cubic, body centered cubic, and face centered cubic are plotted as various types of squares.

\section{DISCUSSION}

That the shape of the grains is an important parameter for the structure is evident from the experiment by Donev et al.
[1], who studied the coordinations in an ensemble of ellipsoids and found a mean coordination number of 9.82 in an ensemble of packing fraction $c=0.685$. This value is much larger than that found in spherical ensembles at similar (but lower) $c$. The compaction of hard spherical grains to higher densities than the random close packed (RCP) value $(c=0.63-0.64)[8,30]$ occurs by increasing the amount of crystalline regions. Pouliquen et al. [6] obtained a strongly crystalline packing $(c=0.67)$ from monodisperse glass spheres by horizontal shaking and slow filling of a container. However, handling hard spheres without fine-tuning the assembling procedure commonly results in values around $c$ $=0.64$. The compaction of deformable grains allows larger packing fractions to be obtained than hard grain ensembles do, because the shape of individual grains can change. Whereas the crystallization of compacting hard grains would show up in the RDF, the evolution of the RDF during compaction is not given in ductile ensembles, as they might compact by structural ordering or grain deformation. Shape changes from that of a sphere or a disk are expected to cause disordering (e.g., there are no crystalline arrangements of pentagons). There are space filling crystalline structures (squares, pyramids), but these are very unstable under any geometrical perturbation, as more than three edges meet at each vertex of the structure.

The radial distribution functions in two and three dimensions as they were studied here were both seen to lose some of the structure associated with hard grain arrangements as high packing fractions were approached. The RDF in 2D numerical hard disk ensembles has been shown [31] to develop its structure considerably through packing fractions $c=0.3-0.83$. The split second, the third, and the fourth peaks were seen to develop between $c=0.7-0.83$, and the RDF at $c=0.83$ resembles the $\mathrm{RDF}$ of the $2 \mathrm{D}$ ductile experiment in the initial stage (Fig. 6). Our 2D ductile system, when further compacted, gives less distinct splitting of peaks, and the RDF thus loses some of its detailed structure toward $c=0.99$ (see Fig. 6). Interestingly, we saw that the peak at $r / d=1.695$ corresponded to a cluster of four grains in the configuration shown in Fig. 6 in the initial part of the experiment, the same configuration that contributes to the peak at $r / d=\sqrt{3}$ in hard disk ensembles. Thus, the initial part of our experiment is dominated by similar configurations as in hard disk ensembles, but with shortened distances. At the end of our experiment, though, several different configurations contribute to this peak in the RDF, thus there are important structural changes compared to hard disk packings.

A similar evolution is expected for 3D ensembles; a certain packing fraction must be reached for locally ordered grain arrangements to occur, and for details in the RDF structure to emerge. When grain deformation becomes important for further compaction, the RDF structure becomes less distinct (Fig. 10). This "destructuring" should become influential in the $3 \mathrm{D}$ ductile grain ensembles at packing fractions close to the random close packing $c \simeq 0.64$ assuming that frictional forces between the grains prevent rearrangements. The RDF of the ductile 3D system at $c=0.70$ has no clear peaks after $r / d=3$, and has much smaller and broader peaks than that of the 2D system at $c=0.99$. This difference could be due to the extra degree of freedom introduced by the third 
dimension, or possibly the differences in rheology between the materials used in $2 \mathrm{D}$ and $3 \mathrm{D}$, and how they react to the way they were compacted. Also a size distribution of the grains would tend to broaden the peaks in the RDF, but the size distribution is similar for our $2 \mathrm{D}$ and $3 \mathrm{D}$ experiments $(3$ and $2.3 \%$, respectively).

The evolution of the coordination number distribution suggests geometrical ordering to take place in the 2D ductile system. The distribution narrows considerably, and only the number of grains with six contacts increases, see Fig. 8. Grains with only two contacts occur for a limited range of packing fractions $c=0.906-0.918$, and these are due to shear motion, during which two neighboring ordered regions align. In three dimensions, the evolution of the coordination number distribution is not as distinct. The average $\langle k\rangle$ increases, but the width of the distribution stays practically constant during compaction. The reason for this could be that the $3 \mathrm{D}$ ductile systems are not as compacted as the $2 \mathrm{D}$ system is, thus a marked narrowing of the coordination number distribution might not take place until larger packing fractions are reached. Also, the mean coordination number in $2 \mathrm{D}$ has a maximum of 6 , while no established maximum exists in 3D. Thus, the distribution of $k$ in 2D must narrow as the system gets denser, whereas the 3D system does not have this strict constraint.

In 2D, the evolution of the average coordination number $\langle k\rangle$ increased with packing fraction $c$ toward the theoretical value $\langle k\rangle=6$, which applies to $2 \mathrm{D}$ space filling structures (Voronoi cells). At the end of the 2D experiment, $1 \%$ of pore space remained, and according to Fig. 9, the increase in average coordination number must be steep for $c$ in the range $0.99-1$. The evolution of $\langle k\rangle$ follows the straight line between the square lattice $(\langle k\rangle=4)$ and the space filling structure $(\langle k\rangle=6)$ up to $c=0.97$. Although an increase of $\langle k\rangle$ with $c$ was expected, the linear evolution was not. The dominant crystalline structure is triangular from the start of the experiment, and regions of dense triangular structures $(k=6)$ were thought to develop rapidly during the initial compaction.

Three-dimensional deformable grains will approach a space filling structure of polyhedra with increasing packing fraction. This structure is not necessarily the packing's Voronoi structure, as the interfaces between neighboring grains then would have to be normal to the center-to-center distance. However, the statistical features of the Voronoi structure might be applicable at $c=1$. No theoretical value exists for the average coordination at $c=1$ in $3 \mathrm{D}$, but a value around 13.6 was found by Bernal [29] based on an experiment with compacted plasticine. The Voronoi tessellation of hard sphere packings at different packing fractions has shown that the average number of sides of a Voronoi cell $\langle f\rangle$ decreases with increasing packing fraction [32,33]. From Voronoi analysis of the ductile ensemble at $c=0.70$, we find that $\langle f\rangle=14.5$, and presume that $\langle f\rangle$ in the ductile ensembles at $c=1$ must be below this value. At a packing fraction of $c=1$, all the faces of the polygon must be touching a neighbor, thus $\langle f\rangle=\langle k\rangle$ at $c=1$. This suggests that had the ductile ensembles been allowed to compact to $c=1$, their average coordination number would be less than 14.5 , and possibly close to Bernal's experimental value of 13.6.
How the Voronoi cell structure changes during compaction must depend on the compaction procedure. If the packing is compacted isomorphically, the relative positions of the grains are fixed with respect to each other, and the Voronoi structure is constant. For grains on the square lattice, the Voronoi cells would remain squares throughout an isomorphic compaction, thus $\langle k\rangle=4$ for all $c$. A random initial packing, or a nonisomorphic compaction procedure, would instead result in an increase of the average coordination number toward $\langle f\rangle$. Thus the trajectories a compacting system makes in the $(\langle k\rangle, c)$ plane is not fixed, but is expected to depend on initial configuration and compaction procedure. As seen from Fig. 12, the ductile Play-Doh ensemble compacts in a self-compacting trajectory (apart from the last point on the curve), which evolves differently than the compacted bronze powder. The bronze powder was compacted by increasing uniaxial pressure, while our system sustained constant uniaxial pressure of gravity. Also, the Rampage model of compaction has a trajectory leading into the region where the ductile compaction starts, but has a different slope at $c=0.64$ than the trajectory of the ductile packings. The different compaction procedures might be an explanation for these different trajectories in the $(\langle k\rangle, c)$ plane.

Hard spherical ensembles can rather easily be compacted to the RCP value $c \simeq 0.64$ and are well represented by Finney's packing in the $(\langle k\rangle, c)$ plane. This point might be fairly common for spherical ensembles, considering that both the Rampage model and two of the ductile ensembles plot in the immediate neighborhood. Looser initial configurations might be obtained in packings of high cohesion or friction, which would change the starting point of the compaction in the $(\langle k\rangle, c)$ plane. That the bronze powder of Ref. [14] plots at a higher $\langle k\rangle$ than the RCP is thought to be due to the wide size distribution of grains, and the sintering procedure used. The hard elliptical grain ensemble [1] was assembled in the same way as the Finney packing, but produced a much higher packing fraction and average coordination number. The ability of the grains in the ductile packing to change their shape does not, however, take the ductile packing into the ellipsoid value of $\langle k\rangle=9.8$ until $c>0.75$ for the Play-Doh ensembles, and $c \sim 0.9$ for the bronze powder (see Fig. 12). Hence, the initial grain shape is an important factor for the initial compaction state in the $(\langle k\rangle, c)$ plane.

\section{CONCLUSION}

We have performed compaction experiments on two- and three-dimensional packings of monodisperse ductile grains, and studied the evolution of their structure with increasing packing fraction. The radial distribution function and coordination number distribution were found for both systems. The radial distribution function developed broader and smoother peaks, seemingly losing ordered structure in both $2 \mathrm{D}$ and $3 \mathrm{D}$. The 2D local configuration around grains in distances $r / d=1.695 \pm 0.05$ revealed that while mainly one configuration contributed to the corresponding peak in the initial RDF, two configurations contributed to the same peak at the most compacted stage of the experiment. The coordination number 
distribution narrows considerably in the 2D packing during the compaction, while no such narrowing is seen in the $3 \mathrm{D}$ system. The average coordination number in $3 \mathrm{D}$ evolves beyond that of hard spherical ensembles, and its evolution is discussed in relation to other granular ensembles, as the compaction procedure and initial grain shape seem to be important parameters for the compaction trajectory in the $(\langle k\rangle, c)$ plane.

This is a new approach to the study of compaction in granular ensembles, which emphasizes the importance of grain ductility on the evolving structure. Further work is necessary to understand the importance of compaction proce- dure, initial grain shape, and rheology for the final structure. In particular, 3D in situ experimental investigations of evolving geometry during compaction would give a strong contribution to the understanding of how local structure depends on compaction procedure.

\section{ACKNOWLEDGMENTS}

The project has been supported by the Norwegian Research Council through the Fluid Rock Interaction Strategic University Program (Grant No. 113354-420). L.U. would like to thank Birger Sevaldson for use of the MicroScribe.
[1] A. Donev, I. Cisse, D. Sachs, E. A. Variano, F. H. Stillinger, R. Connelly, S. Torquato, and P. M. Chaikin, Science 303, 990 (2004).

[2] J. Q. Xu, R. P. Zou, and A. B. Yu, Phys. Rev. E 69, 032301 (2004).

[3] A. J. Forsyth, S. R. Hutton, C. F. Osborne, and M. J. Rhodes, Phys. Rev. Lett. 87, 244301 (2001).

[4] L. Vanel, D. Howell, D. Clark, R. P. Behringer, and E. Clément, Phys. Rev. E 60, R5040 (1999).

[5] C. R. Bloomquist and R. S. Shutt, Ind. Eng. Chem. 32, 827 (1940).

[6] O. Pouliquen, M. Nicolas, and P. D. Weidman, Phys. Rev. Lett. 79, 3640 (1997).

[7] J. L. Finney, Proc. R. Soc. London, Ser. A 319, 479 (1970).

[8] J. D. Bernal and J. Mason, Nature (London) 188, 910 (1960).

[9] J. B. Knight, C. G. Fandrich, C. N. Lau, H. M. Jaeger, and S. R. Nagel, Phys. Rev. E 51, 3957 (1995).

[10] P. Ribiére, P. Richard, D. Bideau, and R. Delannay, Eur. Phys. J. E 16, 415 (2005).

[11] P. Richard, P. Philippe, F. Barbe, S. Bourles, X. Thibault, and D. Bideau, Phys. Rev. E 68, 020301(R) (2003).

[12] E. R. Nowak, J. B. Knight, M. L. Povinelli, H. M. Jaeger, and S. R. Nagel, Powder Technol. 94, 79 (1997).

[13] J. M. Weller, Am. Assoc. Pet. Geol. Bull. 43, 273 (1959).

[14] H. Fischmeister, E. Arzt, and L. Olsson, Powder Metall. 21, 179 (1978)

[15] S. Torquato, T. M. Truskett, and P. G. Debenedetti, Phys. Rev. Lett. 84, 2064 (2000).

[16] T. M. Truskett, S. Torquato, S. Sastry, P. G. Debenedetti, and F. H. Stillinger, Phys. Rev. E 58, 3083 (1998).
[17] J. Feder, J. Theor. Biol. 87, 237 (1980).

[18] J. J. Gonzalez, P. C. Hemmer, and J. S. Hoye, Chem. Phys. 3, 228 (1974).

[19] Y. Pomeau, J. Phys. A 13, L193 (1980).

[20] R. H. Swenden, Phys. Rev. A 24, 504 (1981).

[21] E. L. Hinrichsen, J. Feder, and T. Jøssang, Phys. Rev. A 41, 4199 (1990).

[22] S. Ostojic, E. Somfai, and B. Nienhuis, Nature (London) 439, 828 (2006).

[23] W. K. Pratt, Digital Image Processing (Wiley, New York, 1991).

[24] MATLAB 7.0.4.352 (R14) Library, The MathWorks Inc., 2005.

[25] G. Mason, Nature (London) 217, 733 (1968).

[26] S. Torquato, Annu. Rev. Mater. Res. 32, 77 (2002).

[27] H. S. M. Coxeter, Introduction to Geometry (Wiley, Toronto, 1969).

[28] L. J. H. Alberts, Ph.D. thesis, Delft University of Technology, 2005, http://www.library.tudelft.nl/delftdiss/pdf/2005/ ceg_alberts_20051012.pdf

[29] J. D. Bernal, in Liquids: Structure, Properties, Solid Interactions, edited by T. J. Hughel (Amsterdam, Elsevier, 1965), pp. $25-50$.

[30] G. D. Scott, Nature (London) 194, 956 (1962).

[31] V. Myroshnychenko and C. Brosseau, Phys. Rev. E 71, 016701 (2005).

[32] R. Y. Yang, R. P. Zou, and A. B. Yu, Phys. Rev. E 65, 041302 (2002).

[33] L. Oger, A. Gervois, J. P. Troadec, and N. Rivier, Philos. Mag. B 74, 177 (1996). 